\title{
A novel 3D Carousel based on Pseudo-Haptic Feedback and Gestural Interaction for Virtual Showcasing
}

\author{
Pierre Gaucher* \\ Orange Labs Rennes
}

\author{
Ferran Argelaguet ${ }^{\dagger}$ \\ Inria Rennes
}

\author{
Jérôme Royan \\ Orange Labs Rennes
}

\author{
Anatole Lécuyer ${ }^{\S}$ \\ Inria Rennes
}
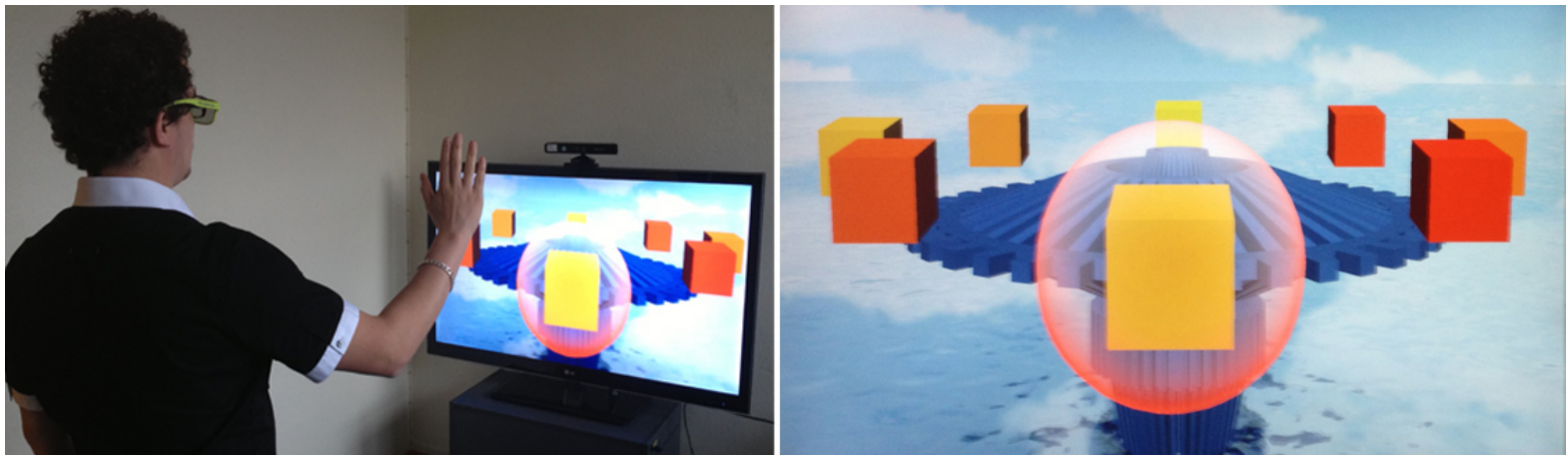

Figure 1: (Left) manipulation of our carousel based on gestural interaction, (Right) screenshot of our 3D carousel application

\begin{abstract}
This paper describes a novel 3D carousel intended for virtual showcasing. The carousel is based on a 3D ring menu which is rendered on a 3D display. The user interaction with the carousel is achieved by tracking the user's gestures while the behavior of the carousel is modified through physically-based pseudo-haptic effects. The pseudo-haptic effect is based on modifying the friction coefficient taking into account the relevance of the objects displayed in the carousel. In addition a magnetic effect is introduced in order to attract to the active item of the carousel. The results from a first user study are globally encouraging and provide insights about the potential of our 3D pseudo-haptic carousel for virtual showcasing.
\end{abstract}

Index Terms: H.5.2 [Information Interfaces and Presentation]: User Interfaces Interaction Styles-; I.3.7 [Computer Graphics]: Three-Dimensional Graphics and Realism Virtual reality-

\section{INTRODUCTION}

In this paper we focus on the use of low-cost VR technologies to showcase virtual products. Indeed, many application fields aim at presenting content using ground-breaking showcase. From professional exhibitions, to virtual shopping, or art installation in a museum, interactive kiosks can greatly improve the experience of a large public.

Our goal is thus the development of an interactive kiosk for the showcasing of virtual products. The requirements of such a widespread application include a minimum training process, a natural and intuitive user interface. Among the large number of existing 3D graphical user interfaces (see survey from Dachselt and Hübner [3]), we chose ring menus and $3 \mathrm{D}$ carousels. These interfaces are

\footnotetext{
*e-mail: pierre.gaucher@orange.com

†e-mail: fernando.argelaguet@inria.fr

†e-mail: jerome.royan@orange.com

$\S$ e-mail: anatole.lecuyer@inria.fr
}

already well-known and are widely used to navigate in multimedia catalogs (web, music, image, video, 3D models). Furthermore, in order to increase the immersion of the user without the need of expensive equipment, our choice was the use of marker-less tracking and a passive stereoscopic display. The consistency between 3D gestural interaction and 3D visual content is expected to improve usability as the user will interact directly with 3D content using his or her own body. The user will only require to wear polarized glasses.

Therefore, the main contributions of this work are : (1) the design of a control law for virtual carousels based on users hand gestures, (2) the extension of the proposed control law to introduce a pseudo-haptic feedback meant to repulse/attract the user to some specific items, and (3) a user evaluation of the proposed approach.

In the remainder of the paper we first describe some related work in 3D graphical user interfaces and pseudo-haptics. Second, we detail the different components of our approach focusing on our interaction metaphors and new pseudo-haptic effect. Finally, we present the results of a user study and end up with some concluding remarks.

\section{Related wORK}

Many previous research works in VR and AR fields have proposed new interfaces for showcasing. They are generally based on relevant relationships between the user interactions, the display device and the 3D representation. As an example, Bimber et al. [2] proposed an AR-based virtual showcase well-suited to supply museum visitors with additional information on artifacts. Hachet et al. [6] also proposed innovative ways to showcase and interact with 3D content in multi-user scenarios. Furthermore, Hachet et al. [5] also proposed handling hand occlusions with adapted graphical interfaces and using a raised transparent projection screen.

Concerning graphical user interfaces, and more precisely ring menus introduced by Liang [7] and carousels, previous research focused on facilitating carousel manipulation with adapted interaction devices, such as the wand [4]. Other studies [10] focus on the issue of showing a large number of items around the carousel without interfering with the usability and use variable distribution in order to reduce the density of items on the front. 


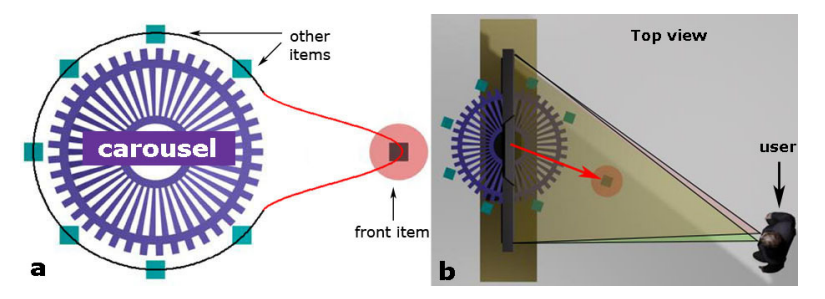

Figure 2: (a) Distortion of the carousel according to a drop shape to display the preselected object closer to the user, (b) attraction of the target item in user direction

More recently, the Microsoft Kinect is being used in many demonstrations by the Kinect-hack community [1]. These low-cost interaction devices present the advantage of not requiring to wear them. On the other hand, they do not provide users with any haptic feedback which are of real interest for a consistent interaction.

Pseudo-haptic feedback is a recent technique meant to simulate haptic sensations by playing with visual feedback [8]. Pusch et al. [9] studied a novel pseudo-haptic effect through a method called HEMP which provides haptic-like sensations by dynamically displacing the visual representation of the user's hand. Promising results confirms that pseudo-haptic effect can be used in gestural and natural interaction.

\section{A NOVEL 3D CAROUSEL WITH PSEUDO-HAPTIC EFFECT}

In this section we describe the components of our showcase system. We detail the visual requirements, the interaction metaphors used and the control laws of the proposed 3D carousel.

\subsection{Visual display}

The 3D carousel is displayed on a stereoscopic screen requiring only the use of passive glasses. According to the size of the screen, the size of the carousel can be adjusted to maximize its size. Head tracking is provided through a Microsoft Kinect placed on top of the screen (see Figure 3.a), enabling the user to explore the carousel from different viewpoints.

When the user is interacting with the carousel, the active item is displaced towards the user by distorting the carousel according to a drop shape (see Figure 2.a). In order to avoid the clipping of an active item with the sides of the viewing frustum, the drop shape always points to the user's head position. Thus, we ensure that the virtual object always remains within the frustum (see Figure 2.b).

\subsection{Gestural interaction}

The gestural interactions involved in our application rely on two metaphors: a 3D carousel for object selection and two-handed manipulation to explore the selected object. In order to rotate the carousel, the user has to perform swipe gestures. When the user performs a right swipe with the left hand, the carousel rotates counterclockwise and when the user achieves a left swipe with the right hand it rotates clockwise. To select an item, the user moves both hands forward (see Figure 3.b), leading to the examination mode. In the examination mode, the user can translate, rotate and scale the selected object using both hands (see Figure 3.c). The transformation applied to the object is computed according to the $3 \mathrm{D}$ vector defined by the position of both hands. All there gestural interactions can be found in previous 3DUI and were carefully chosen to drive the manipulation of the 3D carousel. But the main innovation of our work relies on the physically-based pseudo-haptic effect, which is detailed in the following sections.

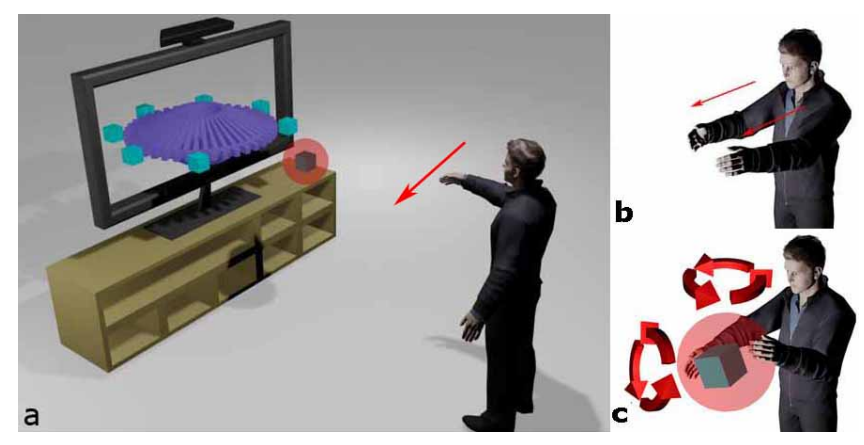

Figure 3: Gestural interaction to control the 3D carousel and manipulate items: (a) global view of the scene, one-hand motion to turn the carousel, (b) two-hand gesture to select an item, (c) two-hand manipulation of the virtual ball.

\subsection{Physical control law}

The behavior of the carousel is controlled by a physical law in order to provide the user with natural and realistic visualization and interaction capabilities. The carousel has a constant friction coefficient $\left(k_{c}\right)$ and a mass $\left(m_{c}\right)$. According to the current tangential speed of the carousel $\left(\mathbf{S}_{c}\right)$ and the speed of the swipe gesture $\left(\mathbf{S}_{h}\right)$, we compute the friction force of the carousel $\left(\mathbf{F}_{c}\right)$ and the force applied to the carousel by the user $\left(\mathbf{F}_{h}\right)$ (see Equations 1 and 2). Variable $k_{h}$ is the friction coefficient between the hand and the carousel:

$$
\begin{array}{r}
\mathbf{F}_{h}=k_{h}\left(\mathbf{S}_{h}-\mathbf{S}_{c}\right)^{2} \\
\mathbf{F}_{c}=-k_{c} \mathbf{S}_{c}^{2}
\end{array}
$$

By applying Newton's second law, we compute the new acceleration of the carousel $\left(a_{c}\right)$ :

$$
a_{c}=\frac{\mathbf{F}_{h}+\mathbf{F}_{c}}{m_{c}}
$$

Finally, we update the new speed of the carousel $\left(\mathbf{S}_{c, t+1}\right)$ taking into account the time increase $(\Delta t)$ :

$$
\mathbf{S}_{c, t+1}=\mathbf{S}_{c, t}+\Delta t \cdot a_{c}
$$

Therefore, the user can control the rotation speed of the carousel by moving the hand more or less quickly. Moreover, when the user hand is not interacting with the carousel, it keeps its inertia and slows down progressively until it stops. For the experiments we used the following values: $m_{c}=10, k_{c}=10$ and $k_{h}=30$.

\subsection{Magnetic Attraction}

The carousel behavior defined so far does not ensure that when the carousel stops there is an object in front of the user (it might stop in the gap between two items). To avoid this behavior, we introduce a magnetic force to the system $\left(\mathbf{F}_{m}\right)$ which will center the current item in front of the user. The magnitude of the force depends on the distance (D) between the current item and the attraction point.

$$
\mathbf{F}_{m}=\frac{1}{D^{2}}
$$

The magnetic effect is only enabled when the speed of the carousel is below a certain threshold and the user is not interacting with the carousel. 

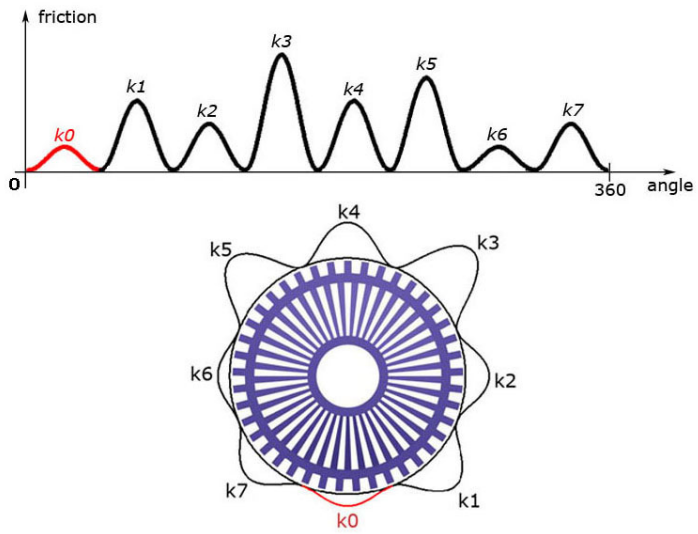

Figure 4: Pseudo-haptic effect: Sliced carousel with varying friction coefficients.

\subsection{Pseudo-haptic feedback}

We introduce a novel pseudo-haptic effect to the carousel to highlight relevant items, such as promotional products by locally modifying the friction of the carousel. This effect is expected to "attract" the user towards these specific items when interacting with the carousel.

First, we assign a friction coefficient for each item in the carousel (e.g. higher values for promotional items, see Figure 4). Then, we define a sinusoidal function $\left(k_{i}(\alpha)\right)$ which has its local maxima at the center position of each item; the maximal values are defined by the assigned friction coefficients. The resulting friction coefficient will increase the friction coefficient of the carousel $\left(k_{c}\right)$ modifying the friction force of the carousel $\left(\mathbf{F}_{c}\right)$.

$$
\mathbf{F}_{c}=-\left(k_{c}+k_{i}(\alpha)\right) \mathbf{S}_{c}^{2}
$$

Consequently, when a user is facing an item with a strong friction coefficient, he has to increase the amplitude of the movement of the hand to move to the following or previous item. If the coefficient is great enough, the user will have to perform several swipe gestures to move to next item. On the contrary, if the friction coefficient is smaller, a light movement will rapidly turn the carousel to the next item. Thus a fast movement of the hand will scroll several items with small coefficients.

\section{User Evaluation}

The main purpose of pseudo-haptic feedback is the increase of the expressiveness of the user interface. However the user interface designer might configure the effect achieving slightly different behaviors. In order to explore the limits of the proposed pseudo-haptic feedback, we have performed a user evaluation of the system exploring its usability and how the user experience is modified when interacting with it.

\subsection{Tasks}

The evaluation consisted in two tasks. The first task was a selection task, participants were instructed to select the highlighted item in the carousel. The goal of the first task was to explore whether the pseudo-haptic feedback proposed hinders user's performance when selecting items in the carousel. In contrast, the second task was an exploration task. Participants had to find which object in the carousel had a "different" behavior, all items in the carousel had the same visual appearance. Participants were instructed to explore the items in the carousel in order to locate the item which had a different and singular behavior. After 30 seconds of exploration if the user is not able to find the singular item, the trial is considered as missed. The goal of the second task was exploring whether the users were able to perceive the pseudo-haptic feedback.

\subsection{Design}

The independent variables for the first (selection) task were, all within-subject factors with repeated measures, (1) the Magnetic effect (enabled, disabled), (2) the friction coefficient $(0,20,80,140)$ and (3) the target to select $(2,4,6)$. Three repetitions were done for each combination. Regarding the target numbering, the target 0 was the one facing the user while the others were numbered clockwise, the carousel used during the evaluation had eight items. The carousel was initialized after each selection trial. The dependent variables were the time required to select the target and the erroneous selections for each trial. In addition, we considered two different configurations of the carousel (A) only the target increases the friction of the carousel and (B) all items except the target increases the friction. Users performed the first with the configuration (A) followed by (B).

For the second task, the independent variables were: the (1) the Magnetic effect (enabled, disabled), (2) the target $(2,3,4,5,6)$ and (3) the friction coefficient $(20,50,80)$. We did not consider the friction coefficient 140 (as in the first task) because during pilot experiments the ratio of recognition was $100 \%$. All within-subject factors, participants did two repetitions for each combination. The dependent variables were the recognition time and the number of wrong recognitions per trial.

For both tasks, the order of the trials was randomized and users were provided a short training to ensure that they understood the procedure. At the end of the experiment users were asked to fill a short questionnaire. User needed around 30 minutes to finish the experiment.

\subsection{Apparatus and Participants}

The evaluation was conducted in a 50" passive stereo TV. The application was developed with Unity 3D and we used Microsoft Kinect to track the user's head and hands. Users interacted with the carousel sitting down, at 2 meters from the TV. Eight users from 21 to 32 years took part in the experiment, 5 male and 3 female. None of them had previously used the system and all users were right handed.

\subsection{Results}

For all post-hoc comparisons, Bonferroni adjustments for $\alpha=95 \%$ were applied; only significant post-hoc comparisons are mentioned $(p<0.05)$

Selection Task Results (see Figure 5). For the configuration A of the carousel, the ANOVA of selection time showed a main effect for the Magnetic condition (M) $\left(F_{1,7}=5.25 ; p<0.05\right)$, the Friction coefficient $\left(F_{c}\right)\left(F_{1,7}=32.03 ; p<0.001\right)$ and the Target $(\mathrm{T})\left(F_{1.7}=\right.$ $15.34 ; p<0.001)$. The ANOVA also showed a two-way interaction between $\mathrm{M}$ and $F_{c}\left(F_{1,7}=2.37 ; p<0.05\right)$. Post-hoc tests showed that users required more time to select an item when $F_{c}=0$. Users also required more time when the magnetic effect was enabled, but only when $F_{c}=0$. Moreover, users required significantly more time to select target 4 . The ANOVA of errors showed a main effect for $F_{c}\left(F_{3,7}=6.83 ; p<0.001\right)$ and an interaction effect between $F_{c}$ and $\mathrm{M}\left(F_{3,7}=2.74 ; p<0.05\right)$. Post-hoc tests showed that users made more mistakes only when $F_{c}=0$ and the magnetic effect was enabled.

For the configuration $\mathrm{B}$, the ANOVA showed a main effect of $F_{c}\left(F_{3.7}=160.43 ; p<0.001\right)$ and $\mathrm{T}\left(F_{2,7}=55.46 ; p<0.001\right)$. Post-hoc tests showed that as the friction increases the selection time also increases except for $F_{c}=20$ which did not showed 


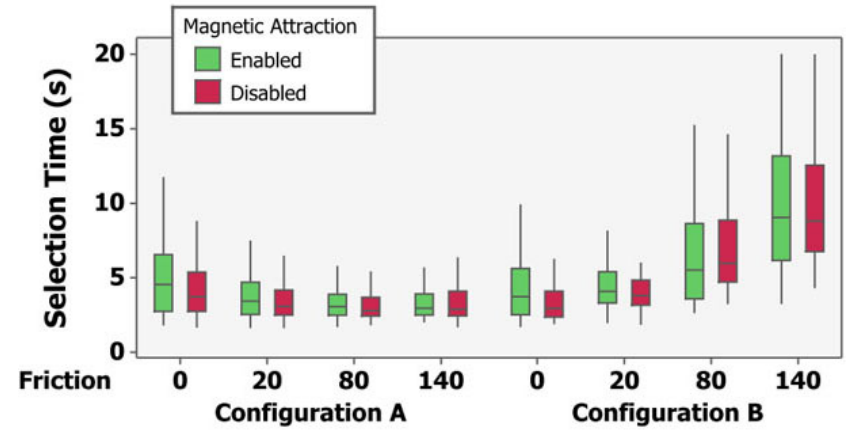

Figure 5: Boxplot of the selection time for the first task of the user study.

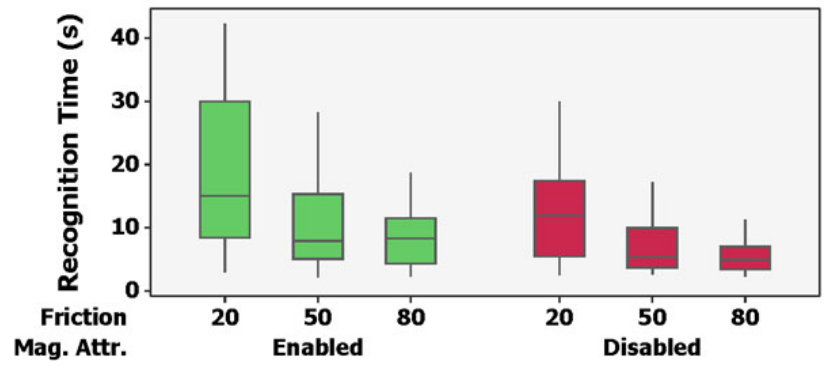

Figure 6: Boxplot of the recognition time when magnetic effect was enabled and disabled.

significant differences with $F_{c}=0$. Similar as the first configuration, Target 4 required more time for its selection. Finally, for the number of errors the ANOVA showed a main effect of $F_{c}$ $\left(F_{3,7}=160.43 ; p<0.001\right)$ and $\mathrm{M}\left(F_{1,7}=5.88 ; p<0.05\right)$. Post-hoc tests showed that users made significantly more mistakes when the Magnetic effect was enabled and when $F_{c}=140$.

Perception Task Results (see Figure 6). The ANOVA of the recognition time, showed a main effect for $\mathrm{M}\left(F_{1,7}=29.51 ; p<\right.$ $0.001), F_{c}\left(F_{2,7}=48.13 ; p<0.001\right)$ and $\mathrm{T}\left(F_{4,7}=2.83 ; p<0.05\right)$. Post-hoc tests showed that users required significantly more time to find the target when the magnetic effect was enabled. Users also required significantly more time to recognize the target when $F_{c}=20$. Moreover, users needed significantly more time to find Target 6 than Target 5. Regarding the number of errors, the ANOVA showed a main effect for $\mathrm{M}\left(F_{2,7}=9.67 ; p<0.001\right)$ and $F_{c}\left(F_{1,7}=5.73 ; p<\right.$ $0.05)$. Users made significantly more errors when the magnetic effect was enabled and for the lowest friction coefficient.

\subsection{Discussion}

From the data gathered from the first task, we can state that the selection of isolated targets is improved, both in selection performance and error rates, when we slightly increase its friction (we got positive results for $F_{c}=20$ ). Moreover, the combination of the magnetic effect when $F_{c} \neq 0$ did not resulted in a drop of performance. In addition, for the condition (B), the usage of the magnetic effect did not introduce any noticeable drawback, but high values of $F_{C}$ resulted in a loss on performance.

Regarding the second task, the perception of the pseudo-haptic effect was better noticed when the magnetic effect was disabled and when the amount of the friction was higher. This is supported by the fact that users required less time and made less misclassifications when the magnetic effect was disabled. As the magnetic effect is triggered when the speed of the carousel is below of a certain threshold, when the magnetic effect was activated, the active item

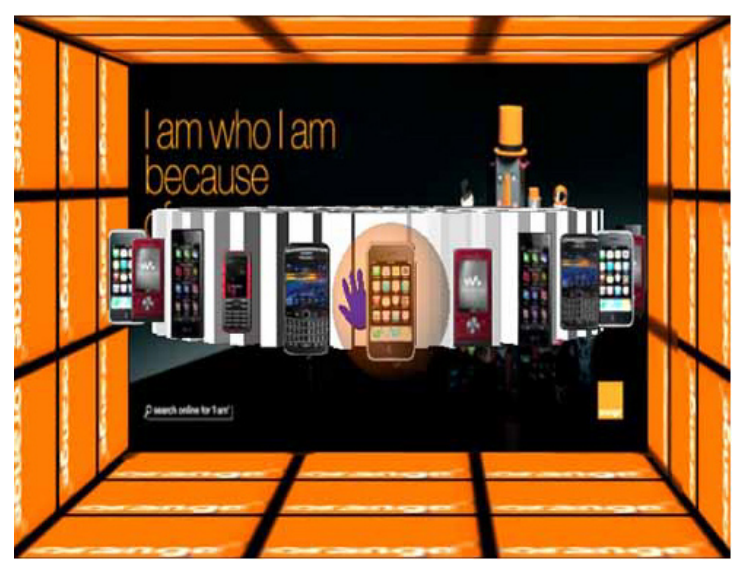

Figure 7: Example application: virtual showcasing of mobile phones.

was confounded with an object with higher friction. This poses the question whether the usage of the both effects at the same time is appropriate. Regarding the perceived friction, we have seen that at low values, although some users are still able to perceive, the effect is too subtle. More experiments should now be conducted to better explore the perception limits.

\section{CONCLUSION}

We have presented a 3D pseudo-haptic carousel interface for virtual showcasing purposes. The virtual products are presented using a 3D carousel augmented with physical behavior and a pseudo-haptic effect aiming to attract the user to specific items. The user, through simple gestures, controls the rotation of the carousel, and can select, examine and manipulate the objects presented. The user study conducted gathered interesting insights about the usability of our system. The gestural interactions seem relatively simple and fast to learn. Users were able to well perceive the pseudo-haptic effects. But the pseudo-haptic effect might decrease the usability of the system if not used properly. The overall application received a positive and enthusiastic first impression. As shown in Figure 7 these promising results suggest novel interaction interfaces for virtual showcasing of items such as telecommunication products.

\section{REFERENCES}

[1] Kinect hacks community. http://kinecthacks.net/.

[2] O. Bimber, B. Fröhlich, D. Schmalstieg, and L. M. Encarnação. The virtual showcase. In Proceedings of ACM SIGGRAPH, 2006.

[3] R. Dachselt and A. Hubner. Three-dimensional menus: A survey and taxonomy. In Computers \& Graphics, 2007.

[4] D. Gerber and D. Bechmann. Design and evaluation of the ring menu in virtual environments. In Immersive Projection Technologies, 2004.

[5] M. Hachet, B. Bossavit, A. Cohé, and J.-B. de la Rivière. Toucheo: multitouch and stereo combined in a seamless workspace. In Proceedings of ACM symposium on User Interface Software and Technology, 2011.

[6] M. Hachet, R. Watanabe, and Y. Kitamura. A collaborative interface for illusionhole using a control-ring and mice. In Proceedings of IEEE symposium on 3D User Interfaces, 2006.

[7] M. G. J. Liang. Jdcad: A highly interactive 3d modeling system. 1994.

[8] A. Lécuyer. Simulating haptic feedback using vision: A survey of research and applications of pseudo-haptic feedback. In Presence: teleoperators and virtual environements, 2009.

[9] A. Pusch, O. Martin, and S. Coquillart. HEMP-Hand-DisplacementBased Pseudo-Haptics: a study of a force field application. In Proceedings of IEEE symposium on 3D User Interfaces, 2008.

[10] S. Wang, M. Poturalski, and D. Vronay. Designing a generalized 3d carousel view. In $\mathrm{CHI}$ 'O5 Extended Abstracts on Human Factors in Computing Systems, 2005. 